\title{
BMJ Open Cardiac arrest after pulmonary aspiration in hospitalised patients: a national observational study
}

\author{
Malin Albert (D) , ${ }^{1}$ Johan Herlitz, ${ }^{2}$ Araz Rawshani (D) , ${ }^{3}$ Mattias Ringh, ${ }^{4}$ \\ Andreas Claesson, ${ }^{4}$ Therese Djärv, ${ }^{5}$ Per Nordberg ${ }^{4}$
}

To cite: Albert M, Herlitz J, Rawshani A, et al. Cardiac arrest after pulmonary aspiration in hospitalised patients: a national observational study. BMJ Open 2020;10:e032264. doi:10.1136/ bmjopen-2019-032264

- Prepublication history and additional material for this paper are available online. To view these files, please visit the journal online (http://dx.doi. org/10.1136/bmjopen-2019032264).

Received 14 June 2019 Revised 02 January 2020 Accepted 28 January 2020

\section{Check for updates}

(C) Author(s) (or their employer(s)) 2020. Re-use permitted under CC BY-NC. No commercial re-use. See rights and permissions. Published by BMJ.

${ }^{1}$ Department of Clinical Science and Education, Sodersjukhuset, Karolinska Institutet, Stockholm, Sweden

${ }^{2}$ Centre for Prehospital

Research, Faculty of Caring Science, Work Life and Social Welfare, University of Borås, Borås, Sweden

${ }^{3}$ Department of Molecular and Clinical Medicine, Institute of Medicine, Sahlgrenska academy, Gothenburg, Sweden

${ }^{4}$ Department of Medicine, Center for Resuscitation Science,

Karolinska Institutet, Stockholm, Sweden

${ }^{5}$ Department of Medicine Solna, Karolinska Institutet, Stockholm, Sweden

Correspondence to

Dr Malin Albert;

malin.albert@sll.se

\section{ABSTRACT}

Objective To study characteristics and outcomes among patients with in-hospital cardiac arrest (IHCA) due to pulmonary aspiration.

Design A retrospective observational study based on data from the Swedish Registry of Cardiopulmonary Resuscitation (SRCR).

Setting The SRCR is a nationwide quality registry that covers $96 \%$ of all Swedish hospitals. Participating hospitals vary in size from secondary hospitals to university hospitals.

Participants The study included patients registered in the SRCR in the period 2008 to 2017. We compared patients with IHCA caused by pulmonary aspiration $(n=127)$, to those with IHCA caused by respiratory failure of other causes $(n=2197)$.

Primary and secondary outcome measures Primary outcome was 30-day survival. Secondary outcome was sustained return of spontaneous circulation (ROSC) defined as ROSC at the scene and admitted alive to the intensive care unit.

Results In the aspiration group $80 \%$ of IHCA occurred on general wards, as compared with $63.6 \%$ in the respiratory failure group $(p<0.001)$. Patients in the aspiration group were less likely to be monitored at the time of the arrest $(18.5 \%$ vs $38 \%, p<0.001)$ and had a significantly lower rate of sustained ROSC ( $36.5 \%$ vs $51.6 \%, p=0.001)$. The unadjusted 30-day survival rate compared with the respiratory failure group was $7.9 \%$ versus $18.0 \%$, $\mathrm{p}=0.024$. In a propensity score analysis (including variables; year, age, gender, location of arrest, initial heart rhythm, ECG monitoring, witnessed collapse and a previous medical history of; cancer, myocardial infarction or heart failure) the OR for 30-day survival was $0.46(95 \%$ Cl 0.19 to 0.94 ).

Conclusions In-hospital cardiac arrest preceded by pulmonary aspiration occurred more often on general wards among unmonitored patients. These patients had a lower 30-day survival rate compared with IHCA caused by respiratory failure of other causes.

\section{INTRODUCTION}

In-hospital cardiac arrest (IHCA) is the most serious complication of critical illness among hospitalised patients. In Sweden in 2017 the incidence of IHCA was 1.7 per 1000 hospital admissions. ${ }^{1}$ Among patients with IHCA

\section{Strengths and limitationsof this study}

- There are few prior studies that have investigated the incidence, setting and consequences of in-hospital cardiac arrests (IHCA) after pulmonary aspiration.

- This observational study is based on data from a well-established nationwide cardiac arrest registry that covers the vast majority of hospitals in Sweden.

- Pulmonary aspiration as the aetiology of the IHCA is not a predefined aetiology in the Swedish Registry of Cardiopulmonary Resuscitation and may be variably reported, which may lead to underreporting and a potential selection bias to the cases where the aspiration was particularly severe.

- The finding from this retrospective register study should be verified in prospective studies.

- Because of the rapid and dynamic nature of resuscitation, reporting of events and timing during resuscitation procedures are typically estimates.

where advanced cardiac life support (ACLS) is initiated the survival rate has been reported to range between $17 \%$ to $30 \% \cdot{ }^{1-4}$ Initial heart rhythm has been reported to be the strongest predictor of survival, with a survival rate of about $50 \%$ if the initial rhythm is shockable, that is, ventricular fibrillation or pulseless ventricular tachycardia and about $10 \%$ if the initial rhythm is non-shockable, that is, asystole or pulseless electrical activity. ${ }^{5-8}$ Previous studies have also identified a higher survival rate when the IHCA occurred in the intensive care unit (ICU), cardiology care units (CCU) or in the cardiac catheterisation laboratory as compared with on general wards $(60 \%$ vs $20 \%) .{ }^{910}$

Although difficult, it is of great importance to establish the cause of the cardiac arrest since the aetiology and its early recognition affect treatment and outcome. ${ }^{1112}$ In one study around $10 \%$ of the IHCA patients reportedly had an associated episode of regurgitation of gastric content, although the authors did not differentiate between pulmonary aspiration 
as a cause of the cardiac arrest or as an outcome. ${ }^{13}$ In out-of-hospital cardiac arrest (OHCA) pulmonary aspiration of gastric content during the course of resuscitation has been reported in $20 \%$ to $30 \%$ of the patients. However, most often it is not reported whether the aspiration occurred prior, during or after cardiopulmonary resuscitation. ${ }^{14}{ }^{15}$ In contrast to OHCA, IHCA patients are under a greater degree of observation and the patient's condition prior to the cardiac arrest can often be diagnosed. Clinical deterioration is common prior to cardiac arrest and many IHCAs are considered preventable in retrospective reviews. ${ }^{16}$ There are few studies assessing the incidence and outcome for IHCA patients with an aetiology of pulmonary aspiration. Therefore, the aims of this study were to compare 30-day survival rate and rate of sustained return of spontaneous circulation (ROSC) in patients with IHCA with an aetiology of pulmonary aspiration of gastric content with IHCA patients with an aetiology of respiratory failure due to other causes.

\section{METHODS}

\section{Study design and ethics}

This is a retrospective observational study based on data from the Swedish Registry of Cardiopulmonary Resuscitation (SRCR). Data were collected according to the Utstein template.

\section{Patients}

The study included patients registered in the SRCR from 1 January 2008 to 31 December 2017. We identified patients who suffered IHCA and received ACLS and whose aetiology was identified as either pulmonary aspiration of gastric content (aspiration group) or respiratory failure (respiratory failure group).

\section{The Swedish Registry of Cardiopulmonary Resuscitation}

Registration of IHCA in the SRCR began in 2005. The registry includes all patients who suffer from cardiac arrest and receive ACLS in the hospital premises. The primary aim of the registry is to improve the quality of care among patients who suffer from IHCA and furthermore to study incidence, demographics and predictors of survival among these patients. ${ }^{1}$ During the first year (2005), 10 hospitals participated, since then the number of participating hospitals has increased to include $96 \%$ $(70 / 73)$ of all Swedish hospitals in 2017. The hospitals vary in size from secondary hospitals with fewer than 50 beds to university hospitals with over 1000 beds (range 45 to 1165$){ }^{17}$

\section{Patients and public involvement}

Patients and members of the public were not involved in the design or planning of the study. The SRCR is a national quality registry. All patients suffering an IHCA are enrolled in the registry. Survivors are later informed about their registration and that their participation is voluntary, such that they can withdraw their consent at any time. This is in accordance with Swedish and European regulations. The Swedish Registry of Cardiopulmonary Resuscitation publishes an annual report open to public. Ethical approval is needed in order to use registry data.

\section{Outcome}

The primary outcome was 30-day survival. Secondary outcome included sustained ROSC, defined as ROSC at the scene and admitted alive to the ICU or CCU.

\section{Data collection}

Data from IHCA patients are entered in the SRCR in two steps. A registered nurse or the physician at the scene of the cardiac arrest reports information to the registry about the resuscitation procedures, including place, key time events (eg, time from collapse to alarm, time from collapse to ACLS, etc), initial cardiac rhythm, ECG monitoring, treatment and if the patient received ROSC. In a second step a registered nurse or physician affiliated with the registry fills out part two of the protocol based on the patient's medical records. This part contains information regarding the probable aetiology of the cardiac arrest (ie, clinical condition before the collapse), 30-day survival, previous medical history, neurological function at discharge and post resuscitation care. Data is collected in accordance with the Utstein protocol. ${ }^{18}$

Aetiology of the IHCA in the registry is divided into eight categories: primary arrhythmia, myocardial infarction/ischaemia, hypotension, pulmonary oedema, respiratory failure, other, unknown and unclear. This is a modification after Utstein's recommendations. ${ }^{18}$ When the aetiology of the IHCA is considered to be multifactorial more than one aetiology will be registered. When pulmonary aspiration of gastric content has been registered as the aetiology of the IHCA it has been registered as the category 'other' and specified as pulmonary aspiration in free text. In the registry respiratory failure is defined as: Respiratory failure regardless of aetiology, known before the event of the cardiac arrest. Please note that the respiratory failure does not require ventilator treatment'. The SRCR only reports on patients with IHCA where resuscitation was attempted and patients with a 'Do Not Resuscitate order' are not included in SRCR. Thus prior to the IHCA patients did not have any restriction in care.

\section{Statistical analysis}

Continuous variables were reported as median with IQR or mean with $\mathrm{SD}$, depending on the distribution. The study groups (aspiration group vs respiratory failure group) were compared using the $\chi^{2}$ test for dichotomous variables and the Wilcoxon two-sample test for continuous variables.

A p value $<0.05$ was regarded as significant. Associations were described with ORs and $95 \%$ CIs.

A propensity score for developing IHCA due to aspiration was computed with logistic regression. The propensity 


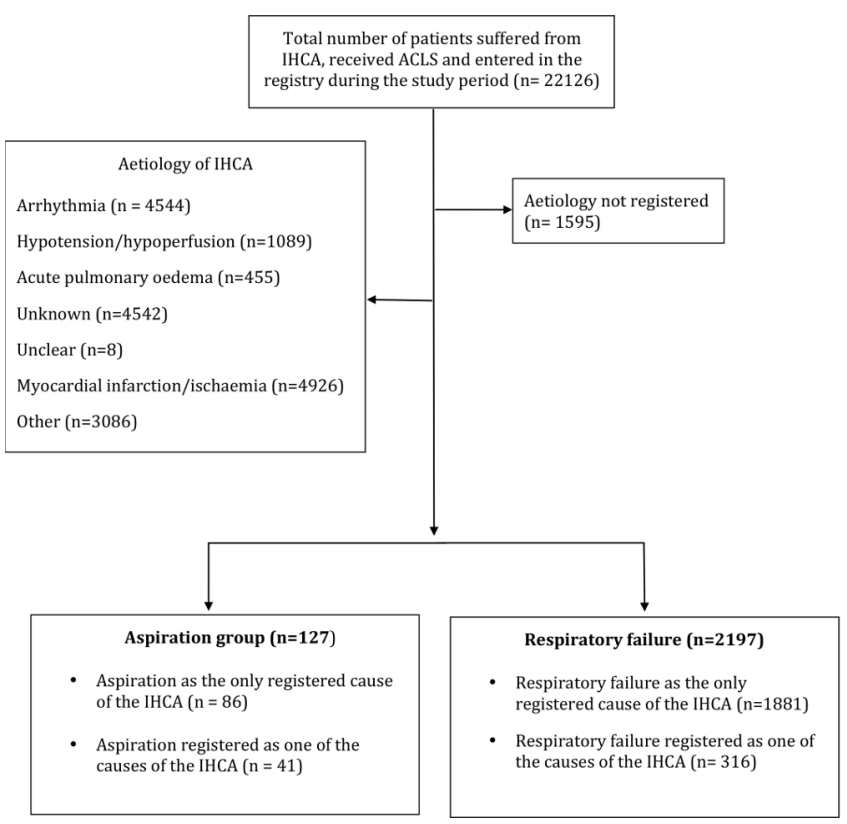

Figure 1 Aetiologies for total number of patients who suffered from in-hospital cardiac arrest (IHCA), received advanced cardiac life support (ACLS) and were registered in the Swedish Registry of Cardiopulmonary Resuscitation (SRCR) during the study period 1 January 2008 to 31 December 2017.

score is the conditional probability of suffering an IHCA due to aspiration, given the characteristics described by the predictors included in the model. We used the propensity score as a covariate in the final model. ${ }^{19}$

The statistical analysis was performed using SAS software V.9.4.

\section{RESULTS}

\section{Baseline characteristics}

During the study period a total of 22126 IHCA patients were reported to the SRCR, the aetiology of the cardiac arrest during the study period was registered for $86 \%$ (20 531 ) of these patients. In all, there were 127 patients in the aspiration group and 1297 patients in the respiratory failure group (figure 1). Of the 127 patients in the aspiration group, 86 had aspiration registered as the only cause of the IHCA. A list of how these patients were registered in the SRCR is presented in the supplement (online supplementary table $\mathrm{S} 1$ ).

Compared with the respiratory failure group, patients in the aspiration group were significantly older, $(74.1 \pm 11.7 \mathrm{vs}$ $69.7 \pm 16.7, p=0.009)$. There were no differences between the groups in terms of sex. Patients in the aspiration group were less likely to have a previous medical history of heart failure (20.5\% compared with $34.6 \%, \mathrm{p}=0.002)$. There were no differences between the two groups in terms of other comorbidities such as previous medical history of myocardial infarction, diabetes, stroke, cancer or renal dysfunction (table 1 ).
Table 1 Baseline characteristics in in-hospital cardiac arrest patients with aetiology of pulmonary aspiration versus respiratory failure

\begin{tabular}{|c|c|c|c|}
\hline & $\begin{array}{l}\text { Aspiration } \\
\text { group }\end{array}$ & $\begin{array}{l}\text { Respiratory } \\
\text { failure group }\end{array}$ & \multirow[b]{2}{*}{$P$ value } \\
\hline & $\begin{array}{l}\mathrm{n}=127 \text { - } \\
\text { missing* }\end{array}$ & $\mathrm{n}=1297-$ missing ${ }^{*}$ & \\
\hline $\begin{array}{l}\text { Age; years, } \\
\text { mean } \pm S D\end{array}$ & $74.1 \pm 11.7$ & $69.7 \pm 16.7$ & 0.009 \\
\hline $\begin{array}{l}\text { Sex; women } \\
(\%)\end{array}$ & $54 / 127$ (42.5) & $930 / 2197$ (42.3) & 1.0 \\
\hline \multicolumn{4}{|c|}{ Previous history, n/N (\%) } \\
\hline $\begin{array}{l}\text { Myocardial } \\
\text { infarction }\end{array}$ & $18 / 125(14.4)$ & $349 / 2128(16.4)$ & 0.62 \\
\hline Heart failure & 24/117 (20.5) & $709 / 2050$ (34.6) & 0.002 \\
\hline Diabetes & $34 / 127$ (26.8) & $571 / 2174(26.3)$ & 0.92 \\
\hline Stroke & $21 / 126(16.7)$ & 257/2173 (11.8) & 0.12 \\
\hline Cancer & $35 / 124$ (28.2) & $465 / 2145$ (21.7) & 0.09 \\
\hline $\begin{array}{l}\text { Renal } \\
\text { dysfunction }\end{array}$ & 69/121 (57.0) & $1213 / 2007$ (60.4) & 0.5 \\
\hline
\end{tabular}

*missing=number of patients where data were missing for specific variable.

\section{Resuscitation characteristics and treatment}

Patients in the aspiration group compared with patients in the respiratory failure group were significantly less likely to have a witnessed IHCA $(67.5 \%$ vs $80.4 \%, \mathrm{p}<0.001)$ or to be monitored at the time of the collapse $(18.5 \%$ vs $38.0 \%, \mathrm{p}<0.001$ ) (table 2 ).

There were no differences between the groups in terms of initial shockable rhythm, use of intubation, epinephrine or mechanical chest compression. IHCA in the aspiration group significantly more often occurred on general wards $(80.2 \%$ compared with $63.6 \%, \mathrm{p}<0.001)$ and significantly less often in the ICU $(2.4 \%$ compared with $11.6 \%$, $\mathrm{p}<0.001)$. There was no difference between the groups in regard to if the IHCA occurred in other high dependant wards such as CCU or cardiac catheterisation laboratory. In terms of key time events there were no differences between the groups with the exception of delay $>3 \mathrm{~min}$ from collapse to defibrillation, which occurred more often in the aspiration group (table 2 ).

\section{Outcomes}

The aspiration group had a significantly lower 30-day survival rate than the respiratory failure group $(7.9 \%$ compared with $18 \%, \mathrm{p}=0.024)$ as well as a lower rate of sustained ROSC (table 3).

In the propensity score analysis the following variables were included: calendar year, age, sex, location of arrest, ECG monitoring, if the collapse was witnessed or not, initial heart rhythm and previous medical history of heart failure, myocardial infarction and cancer. The OR for 30-day survival was 0.46 (95\% CI 0.19 to 0.94$)$ in the 
Table 2 Information of circumstances, location and time of IHCA patients with aetiology of pulmonary aspiration versus respiratory failure

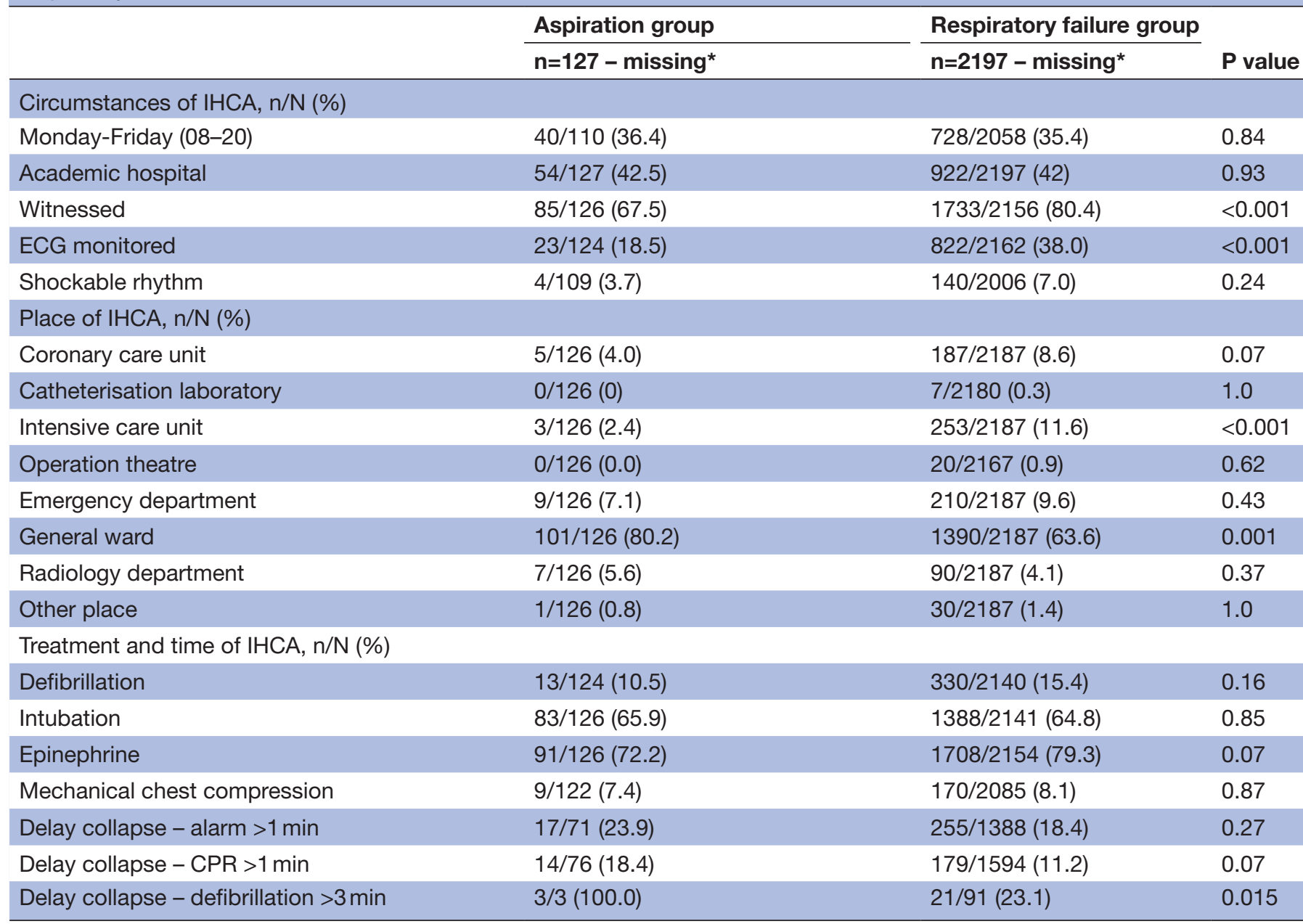

*missing=number of patients where data were missing for specific variable.

CPR, cardiopulmonary resuscitation; IHCA, in-hospital cardiac arrest.

aspiration group compared with the respiratory failure group.

\section{DISCUSSION}

The main findings in this observational study, assessing IHCA due to pulmonary aspiration were that IHCA with the major aetiology of pulmonary aspiration occurred more often on general wards in unmonitored patients, when compared with IHCA with an aetiology of respiratory failure of other causes. When using propensity score to balance the factors before the cardiac arrest, aspiration was associated with a lower 30-day survival rate compared with cardiac arrests caused by respiratory failure of other causes. These findings are important and supports that a higher level of care may be considered in patients with increased risk of pulmonary aspiration to prevent aspiration and potentially subsequent complications such as cardiac arrest.

Many IHCA are considered preventable when evaluated in retrospective reviews. To prevent IHCA the key

Table 3 Outcome of in-hospital cardiac arrest patients with aetiology of pulmonary aspiration versus respiratory failure

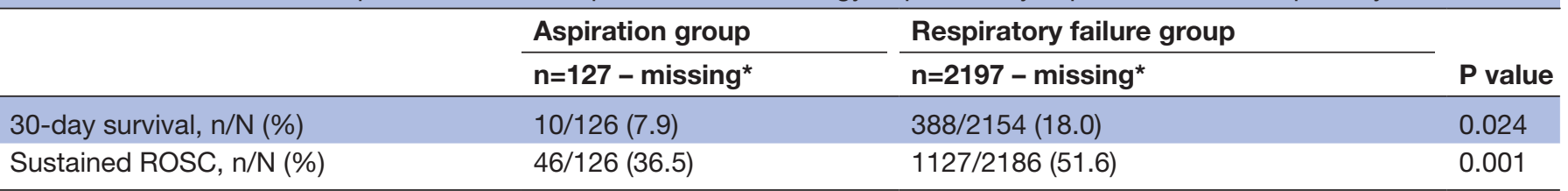

${ }^{*}$ missing=number of patients where data were missing for specific variable.

$\mathrm{ROSC}$, return of spontaneous circulation. 
element is identification of at-risk patients in combination with early interventions to prevent deterioration to cardiac arrest. ${ }^{20}$ If patients with risk of pulmonary aspiration of gastric content can be identified and targeted interventions applied, some IHCA may be prevented. One measure could be to transfer at-risk patients to a higher level of care. In the aspiration group, $80 \%$ of IHCA occurred on general wards compared with $64 \%$ in the respiratory failure group. One explanation to this finding could be that respiratory failure of other reasons may develop over hours, leaving staff more time to recognise these patients and move them to a higher level of care, whereas aspiration generally has a more sudden onset. In addition, several studies have shown that patients who suffer IHCA on general wards, without ECG-monitoring are less likely to have witnessed collapse, a lower probability of an initial shockable rhythm and a lower 30-day survival. ${ }^{10}$ Predictive signs of deterioration and potentially preventable causes of IHCA may be missed on general wards compared with wards with a higher rate of monitoring. ${ }^{10} 2122$ In this cohort which only represented patients with IHCA due to respiratory causes and aspiration, a majority of patients had their collapse on general wards and a minority at the intensive care or other monitored units. These findings regarding where the IHCA occurred are in accordance with previous reports from Sweden and some other European countries, suggesting that about half of cases occur on general wards, about half in monitored wards and about $10 \%$ in intensive care units. ${ }^{1323}$ These proportions may differ from other settings and specific patient populations.

The adjusted analysis on outcome is limited in our study as the number of patients in the aspiration group is small. In the propensity score we have included variables that in previous studies have been shown to affect outcome. ${ }^{1}$ When adjusting for these variables, pulmonary aspiration was associated with a lower 30-day survival rate. We checked the consistency of our results by ${ }^{1}$ computing propensity scores using gradient boosting, a machine learning algorithm, as well as ${ }^{2}$ using logistic regression, without propensity scores, and there were no material differences in the obtained ORs. The unadjusted rate of sustained ROSC was also lower in the aspiration group compared with the respiratory failure group, suggesting that cardiac arrests due to major pulmonary aspiration are more difficult to resuscitate compared with patients with other reasons to severe hypoxia.

The respiratory failure group was significantly younger compared with the aspiration group. Previous studies have reported a clear association between increasing age and poor outcome. ${ }^{23}{ }^{24}$ However, these differences between the groups have been accounted for in the propensity score and the difference in 30-day survival remained.

In a prospective study by Stone et al, the incidence of regurgitation of gastric content prior to IHCA was reported to be about $10 \%$. The authors did not further analyse this data or report the incidence of pulmonary aspiration of gastric content as a cause of the cardiac arrest. In a retrospective study of IHCA by Wallmuller $e t$ $a l$, the proportion of pulmonary aspiration was reported to be about $1 \%$. However, none of these studies investigated the relation between pulmonary aspiration prior to IHCA and outcome. ${ }^{1321}$ In our study the proportion was as low as $0.6 \%$ and the registration of pulmonary aspiration as the aetiology of IHCA in the SRCR is most probably underreported, possibly due to the current system of reporting aetiologies. The potential benefit of identifying patients at risk of pulmonary aspiration and cardiac arrest may therefore be greater than the results that our study suggest.

\section{Strengths and limitations}

This is one of the few studies investigating cardiac arrest after pulmonary aspiration. One of the strengths of this observational study is that the register is well established and covers almost all hospital beds in Sweden. However, there are several limitations to consider. First, the cause of the cardiac arrest may be difficult to establish and may also be multifactorial. Second, pulmonary aspiration as the aetiology of the IHCA is not a predefined aetiology in the SRCR and thus is dependent on the registrar, which most likely contributes to underreporting of cases and those that are registered may have a major aspiration with disproportionately worse outcome. Thus, there is a risk that the present study mainly reports the most severe cases of pulmonary aspiration that lead to cardiac arrest. Third, since the register was introduced in 2005 hospitals have joined over time, which may introduce a selection bias. Fourth, recording and registering the correct time in minutes and seconds is due to the emergency of the situation challenging and it is important to acknowledge that the registered times are estimates. However, this is a non-differential classification error and would in general not contribute to any systematic differences between groups. In addition, in individual cases it may be difficult to assess when the aspiration occurred in relation to the collapse. However, the question that is raised in the registry addresses the clinical condition before and not during or after cardiac arrest.

Many future research challenges remain. There seems to be a knowledge gap with regard to the association between the severity of aspiration and the chance of survival when it is complicated by cardiac arrest. Furthermore, we do not know whether the outcome is different when the aspiration is the major cause of collapse and when it is a contributing factor only. Both these unanswered questions have relevance to the present study in which the research question was to address the cases where aspiration was the major cause of arrest. Finally, we do not know much about the mechanisms by which the aspiration leads to a cardiac arrest and if there are other risk factors than those described in this study that explain the low chance of survival among these patients. These are difficult research questions that will become a challenge to adequately address in the future. 


\section{CONCLUSION}

In this nationwide observational study, IHCA after pulmonary aspiration occurred more often on general wards and in unmonitored patients compared with cardiac arrest caused by respiratory failure of other causes. The 30-day survival rate was significantly lower in patients with cardiac arrest with aetiology of pulmonary aspiration compared with cardiac arrests caused by respiratory failure. Although this study may refer mainly to the most severe cases of aspiration that lead to IHCA, our data highlight the requirement of monitoring these patients and the obvious need to find strategies to prevent aspiration among patients at risk for such a complication.

\section{Twitter Malin Albert @MalinAlbert}

Contributors MA, JH and PN formulated the study question and were responsible for the study concept and design. JH and AR were responsible for study selection, data extraction and statistical analysis. TD obtained ethical approval. MR, AC and TD provided clinical advice. MA wrote the manuscript in consultation with JH and PN. $A R, M R, A C$ and TD reviewed the manuscript and made revision prior to submission. All authors approved the final manuscript.

Funding The authors have not declared a specific grant for this research from any funding agency in the public, commercial or not-for-profit sectors.

Competing interests None declared.

Patient consent for publication Not required.

Ethics approval The study was approved by the Regional Ethical Review Board in Stockholm, Sweden (Identification number 2013/1959-31/4).

Provenance and peer review Not commissioned; externally peer reviewed.

Data availability statement Data is available upon request from the author MA.

Open access This is an open access article distributed in accordance with the Creative Commons Attribution Non Commercial (CC BY-NC 4.0) license, which permits others to distribute, remix, adapt, build upon this work non-commercially, and license their derivative works on different terms, provided the original work is properly cited, appropriate credit is given, any changes made indicated, and the use is non-commercial. See: http://creativecommons.org/licenses/by-nc/4.0/.

\section{ORCID iDs}

Malin Albert http://orcid.org/0000-0002-7346-9796

Araz Rawshani http://orcid.org/0000-0003-2066-3533

\section{REFERENCES}

1 Hessulf F, Karlsson T, Lundgren P, et al. Factors of importance to 30day survival after in-hospital cardiac arrest in Sweden - A populationbased register study of more than 18,000 cases. Int J Cardiol 2018;255:237-42.

2 Girotra S, Nallamothu BK, Spertus JA, et al. Trends in survival after in-hospital cardiac arrest. N Engl J Med 2012;367:1912-20.

3 Nolan JP, Soar J, Smith GB, et al. Incidence and outcome of inhospital cardiac arrest in the United Kingdom national cardiac arrest audit. Resuscitation 2014;85:987-92.

4 Peberdy MA, Kaye W, Ornato JP, et al. Cardiopulmonary resuscitation of adults in the hospital: a report of 14720 cardiac arrests from the National Registry of cardiopulmonary resuscitation. Resuscitation 2003;58:297-308.
5 Tran S, Deacon N, Minokadeh A, et al. Frequency and survival pattern of in-hospital cardiac arrests: the impacts of etiology and timing. Resuscitation 2016;107:13-18.

6 Cooper S, Janghorbani M, Cooper G. A decade of in-hospital resuscitation: outcomes and prediction of survival? Resuscitation 2006;68:231-7.

7 Nadkarni VM, Larkin GL, Peberdy MA, et al. First documented rhythm and clinical outcome from in-hospital cardiac arrest among children and adults. JAMA 2006;295:50-7.

8 Meaney PA, Nadkarni VM, Kern KB, et al. Rhythms and outcomes of adult in-hospital cardiac arrest. Crit Care Med 2010;38:101-8.

9 Adamski J, Nowakowski P, Goryński P, et al. Incidence of inhospital cardiac arrest in Poland. Anaesthesiol Intensive Ther 2016;48:288-93.

10 Herlitz J, Bång A, Aune S, et al. Characteristics and outcome among patients suffering in-hospital cardiac arrest in monitored and nonmonitored areas. Resuscitation 2001;48:125-35.

11 Bergum D, Haugen BO, Nordseth T, et al. Recognizing the causes of in-hospital cardiac arrest--A survival benefit. Resuscitation 2015;97:91-6.

12 Saarinen S, Nurmi J, Toivio T, et al. Does appropriate treatment of the primary underlying cause of PEA during resuscitation improve patients' survival? Resuscitation 2012;83:819-22.

13 Stone BJ, Chantler PJ, Baskett PJ. The incidence of regurgitation during cardiopulmonary resuscitation: a comparison between the bag valve mask and laryngeal mask airway. Resuscitation 1998;38:3-6.

14 Virkkunen I, Kujala S, Ryynänen S, et al. Bystander mouth-to-mouth ventilation and regurgitation during cardiopulmonary resuscitation. $J$ Intern Med 2006;260:39-42.

15 Virkkunen I, Ryynänen S, Kujala S, et al. Incidence of regurgitation and pulmonary aspiration of gastric contents in survivors from outof-hospital cardiac arrest. Acta Anaesthesiol Scand 2007;51:202-5.

16 Galhotra S, DeVita MA, Simmons RL, et al. Members of the medical emergency response improvement team $\mathrm{C}$. mature rapid response system and potentially avoidable cardiopulmonary arrests in hospital. Qual Saf Health Care 2007;16:260-5.

17 Svenska Hjärt- Lungräddningsregistret - årsrapport. Swedish register of cardiopulmonary resuscitation, 2017. Available: https:// registercentrum.blob.core.windows.net/shlrsjh/r/-rsrapport-2017Sy1wvd12Z.pdf

18 Jacobs I, Nadkarni V, Bahr J, et al. Cardiac arrest and cardiopulmonary resuscitation outcome reports: update and simplification of the Utstein templates for resuscitation registries: a statement for healthcare professionals from a task force of the International liaison Committee on resuscitation (American heart association, European resuscitation Council, Australian resuscitation Council, New Zealand resuscitation Council, heart and stroke Foundation of Canada, InterAmerican heart Foundation, resuscitation councils of southern Africa). Circulation 2004;110:3385-97.

19 Austin PC. An introduction to propensity score methods for reducing the effects of confounding in observational studies. Multivariate Behav Res 2011;46:399-424.

20 Andersen LW, Holmberg MJ, Berg KM, et al. In-Hospital cardiac arrest: a review. JAMA 2019;321:1200-10.

21 Wallmuller C, Meron G, Kurkciyan I, et al. Causes of in-hospital cardiac arrest and influence on outcome. Resuscitation 2012;83:1206-11.

22 Sandroni C, Ferro G, Santangelo S, et al. In-Hospital cardiac arrest: survival depends mainly on the effectiveness of the emergency response. Resuscitation 2004;62:291-7.

23 Al-Dury N, Rawshani A, Israelsson J, et al. Characteristics and outcome among 14,933 adult cases of in-hospital cardiac arrest: a nationwide study with the emphasis on gender and age. Am J Emerg Med 2017;35:1839-44.

24 Larkin GL, Copes WS, Nathanson BH, et al. Pre-resuscitation factors associated with mortality in 49,130 cases of in-hospital cardiac arrest: a report from the National Registry for cardiopulmonary resuscitation. Resuscitation 2010;81:302-11. 